\section{Sleep on it!}

Pierre Maquet

Practicing a procedural memory task does not improve
performance untill hours later. Two new studies show that
sleep is absolutely necessary for this memory consolidation.

The function of sleep remains largely unknown, but one prevalent hypothesis is that sleep is involved in reprocessing memory traces and leads to memory consolidation. Two papers in this issue strengthen this hypothesis considerably by providing evidence that sleep, particularly slow wave sleep, is absolutely required for improvement on a visual discrimination task.

To appreciate the advance of these new studies, it is helpful to review what is known about the role of sleep in memory consolidation, and to remember that there are two main types of sleep. Slow wave sleep (SWS) is mostly expressed early in the night and is characterized by large-amplitude, low-frequency electroencephalographic (EEG) oscillations. In contrast, rapid eye movement (REM) sleep increasingly occurs late in the night and is identified by low-amplitude, relatively fast rhythms on EEG recordings, by ocular saccades and by muscular atonia.

In animals learning a new task, subsequent REM sleep increases ${ }^{1,2}$ until the animal has mastered the task ${ }^{1}$. Conversely, performance on various tasks is severely impaired when sleep deprivation follows training sessions in rats ${ }^{1,2}$. Likewise, in humans, increases in REM sleep are reported after training on some tasks (learning Morse code ${ }^{3}$, artificial inversion of the visual field ${ }^{4}$ ), and sleep deprivation impairs performance on recently learned tasks ${ }^{2}$. However, these early studies had two main weaknesses. First, in the human studies, the tasks were not always easily categorized in terms of the now-recognized implicit and explicit memory systems. Second, the sleep deprivation studies did not include sufficient controls-leading to the possibility that impairment could have been due to nonspecific effects of sleep deprivation, such as decreased arousal and motivation and increased stress.

The author is in the Wellcome Department of Cognitive Neurology, The Functional Imaging Laboratory, St. Johns House, 12 Queens Square, London WC1N 3BG, UK.

email:p.maquet@fil.ion.ucl.ac.uk
More recent studies addressed one or both of these criticisms, and two further questions emerged. The first pertains to the differential role of SWS and REM sleep in the processing of implicit and explicit memory traces. Slow wave sleep deprivation seems to particularly impair episodic memories (memories that can be consciously recollected $)^{5}$. In contrast, REM sleep deprivation is specifically deleterious for implicit learning (where performance improvement is not associated with the ability to consciously recall the memory traces underlying the improvement $)^{2,5,6}$. However, this general scheme might be oversimplistic because other data suggest that optimal implicit learning requires sufficient amounts of both SWS and REM sleep ${ }^{7}$, or even the direct succession of SWS by REM sleep periods ${ }^{8}$.

The second question concerns whether sleep is an absolute requirement for memory consolidation-or if sleep only provides favorable conditions for the processing of memory traces. For example, characteristic discharge patterns in hippocampal population recordings (sharp waves and ripples) occur in both SWS and quiet waking ${ }^{9}$. This peculiar pattern of activity, rather than SWS per se, might be sufficient for memory processing. In this view, sleep might have a permissive, non-obligatory, role in memory trace processing.

The two papers in this issue provide key findings related to these two questions.
Both studies tested the implicit learning of a visual discrimination skill ${ }^{6}$. In this task, a target screen is briefly presented, with either a vertical or horizontal array of diagonal bars embedded in one visual quadrant against a background of horizontal bars. The target screen is followed by a blank screen for a variable interstimulus interval (ISI), and then by a mask (a screen filled with V or L letters). The subject is asked to identify the orientation of the array, and his or her performance is estimated by the minimum ISI corresponding to $80 \%$ of correct responses. With this task, improvement is known to require that subjects sleep before retesting (Fig. 1). The two new papers extend this result substantially by demonstrating that the first night after training is crucial for skill acquisition and that both early (mainly SWS) and late (mainly REM) sleep are needed to reach optimal performance.

The paper by Stickgold et al. ${ }^{10}$ suggests that sleeping during the night after a single training session is critical to skill acquisition. Subjects who were sleep deprived during this night showed virtually no performance improvement on the following days. In contrast, subjects allowed to sleep immediately after training showed a significant enhancement in their performance, as early as the first day following training and during the whole ensuing week. The ingenious protocol set up by Stickgold et al. controls for possible indirect deleterious effects of sleep deprivation on performance because the sleep-deprived group was tested after two full nights of recovery sleep (three nights after the training session). These results suggest that the first night of sleep is crucial for acquisition of the new visual skill; sleep after training has an obligatory, not a permissive, role in the consolidation of memory traces.

What happens during this first night

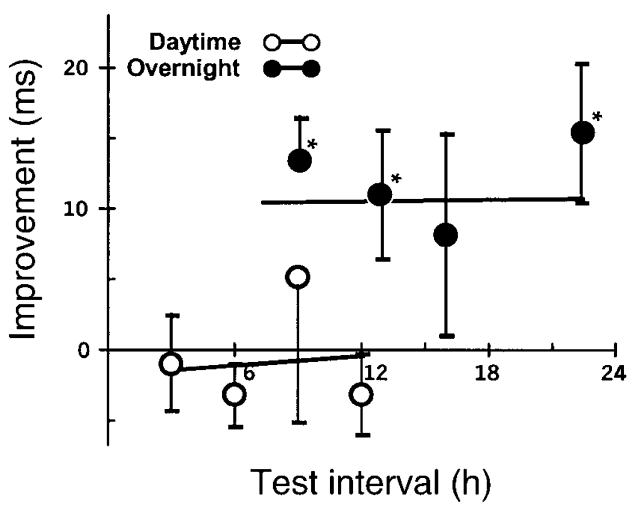

Fig. 1. Improvement on a visual discrimination task was not observed unless subjects obtained at least six hours of sleep between training and retesting. Subjects were tested either on the day of training with no intervening sleep (open circles) or on the day after training, following a night's sleep (filled circles). Means and error bars (s.e.m.) are shown for each test interval. Solid lines are linear regression fits to data points. *Individual groups showing significant improvement. Reprinted with permission from ref. 7. 
after training? Which kind of sleep, SWS or REM sleep, is most important for skill learning? Gais et al. ${ }^{11}$ either trained subjects on the visual discrimination in the evening and retested them after three hours of sleep early in the night (when sleep is mostly SWS), or trained them in the middle of the night and retested them after three hours of sleep late in the night (when REM sleep predominates). In contrast to previous observations ${ }^{6}$, the authors show that performance improved only in the early night condition, when subjects mainly got SWS. In this study also, because of their inventive experimental design and control groups, the authors could rule out any nonspecific effect due to sleep deprivation or to circadian variation. They also observed that the enhancement of performance was even larger when subjects were allowed to sleep the whole night. Gais et al. thus provide the first strong experimental evidence for a two-step process in memory formation during sleep in humans. Given the substantial differences between SWS and REM sleep, it is likely that each sleep stage contributes in a different way to memory trace processing. As stated by the authors, "procedural memory formation would be prompted by early SWS-related processes", whereas "late REM sleep would promote memory formation at a second stage, only after periods of SWS-rich early sleep have occurred".

The cellular mechanisms underpinning memory reprocessing during sleep are still unknown, but experience-dependent changes have been observed at different levels of description in the sleeping brain. At the systems level, functional neuroimaging shows that some brain areas are significantly reactivated in human subjects previously trained on a serial reaction time task (another implicit learning task $)^{12}$. At the cellular level, neuronal re-activations are observed in hippocampal ${ }^{13}$ and cortical ${ }^{14}$ neuronal ensembles during sleep after training. Even at the subcellular level, evidence exists for experience-dependent gene transcription. For instance, the immediate-early gene $z i f-268$ is upregulated during sleep in the cerebral cortex of rats exposed to rich sensorimotor experience in the preceding waking period ${ }^{15}$. Future research will further investigate whether these activity-dependent changes reflect the reprocessing-possibly the consolidation-of memory traces.
1. Hennevin, E., Hars, B., Maho, C. \& Bloch, V. Behav. Brain Res. 69, 125-135 (1995).

2. Smith, C. Behav. Brain Res. 69, 137-145 (1995).

3. Mandai, O., Guerrien, A., Sockeel, P., Dujardin, K. \& Leconte, P. Physiol. Behav. 46, 639-642 (1989).

4. De Koninck, J. \& Prevost, F. Can. J. Psychol. 45, 125-139 (1991).

5. Plihal, W. \& Born, J. Psychophysiology 36, 571-582 (1999).

6. Karni, A., Tanne, D., Rubenstein, B. S., Askenasy, J. J. \& Sagi, D. Science 265, 679-682 (1994).

7. Stickgold, R., Whidbee, D., Schirmer, B., Patel, V. \& Hobson, J. A. J. Cogn. Neurosci. 12, 246-254 (2000).

8. Giuditta, A. et al. Behav. Brain Res. 69, 157-166 (1995).

9. Kudrimoti, H. S., Barnes, C. A. \& McNaughton, B. L. J. Neurosci. 19, 4090-4101 (1999).

10. Stickgold, R., LaTanyoa, J. \& Hobson, J. Nat. Neurosci. 3, 1237-1238 (2000).

11. Gais, S., Plihal, W., Wagner, U. \& Born, J. Nat. Neurosci. 3, 1335-1339 (2000).

12. Maquet, P. et al. Nat. Neurosci3, 831-836 (2000).

13. Wilson, M. A. \& McNaughton, B. L. Science 265, 676-679 (1994).

14. Amzica, F., Neckelmann, D. \& Steriade, M. Proc. Natl. Acad. Sci. USA 94, 1985-1989 (1997).

15. Ribeiro, S., Goyal, V., Mello, C. V. \& Pavlides, C. Learn. Mem. 6, 500-508 (1999).

\section{Keep your eye off the ball}

The frequently heard coach's advice, "Keep your eye on the ball”, may not be the best approach, suggests a new study on page 1236 of this issue. The authors used the cricket batsman's challenge (how to react to a fast-approaching ball) to address how athletes use visual cues to produce rapid and accurate motor responses. Previous theories proposed that a batsman must use direct visual measurements, such as image expansion or the rate of change of binocular disparity, to predict the ball's trajectory. However, given that the batsman has only a fraction of a second to monitor such visual cues, it has been controversial whether these parameters could be measured accurately enough to guide the correct response.

Land and McLeod took a new approach to this problem by monitoring the eye movements of batsmen as the ball was approaching, to determine what type of visual information they were actually collecting. The authors monitored the eye movements of three cricket batsman of

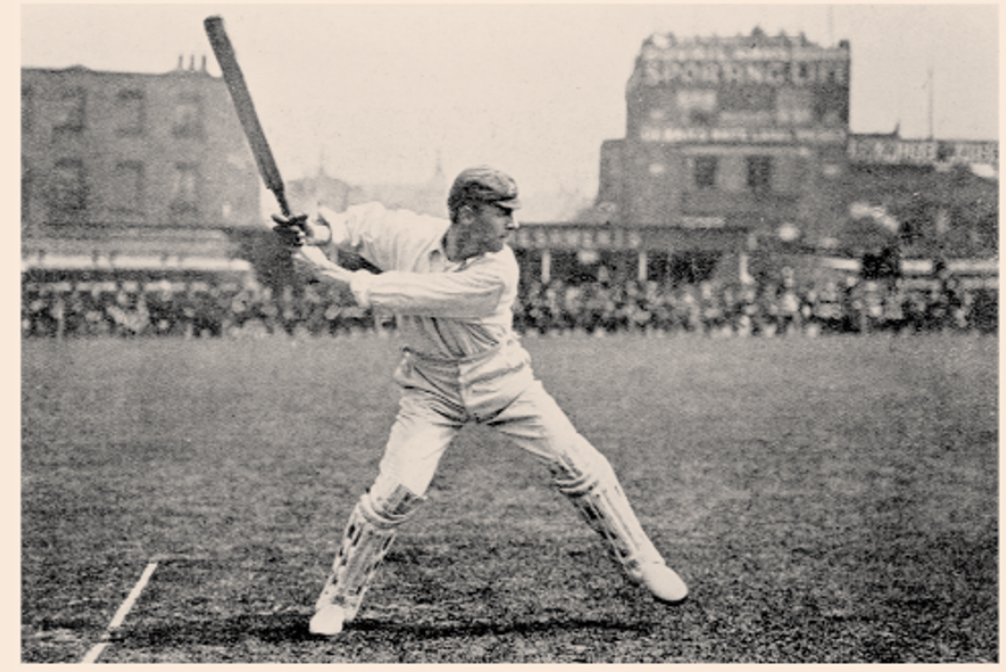
widely varying skill, and found that, in general, they madeasimilar sequence of eyemovements. Their eyes followed the ball's trajectory for a short period after release, then made a saccade below the ball to the site where it would be predicted to bounce. They then fixated again on the ball as it bounced, and followed its upward trajectory for approximately 200 msafterward. The parameters that best distinguished most skilled from least skilled batsmen were the speed and variability of the initial saccade. The best batsman had the shortest delay between the ball's release and saccade initiation, and also used different saccade timing and magnitude for different ball trajectories. Therefore, the cricket bats man prepares his response to an oncoming ball by quickly assessing its predicted trajectory and directing visual attention to appropriateregions of the visual field with a precisely timed sequence of eye movements.

Jack Horne 